TITLE:

\title{
On Solving Semidefinite Programming by Quantifier Elimination
}

$\operatorname{AUTHOR}(\mathrm{S})$ :

ANAI, HIROKAZU

\section{CITATION:}

ANAI, HIROKAZU. On Solving Semidefinite Programming by Quantifier Elimination. 数理解 析研究所講究録 1998, 1038: 154-162

ISSUE DATE:

1998-04

URL:

http://hdl.handle.net/2433/61973

RIGHT: 


\title{
On Solving Semidefinite Programming by Quantifier Elimination
}

\author{
HIROKAZU ANAI \\ e-mail:anai@flab.fujitsu.co.jp \\ High Performance Computing Research Center, \\ FUJITSU LABORATORIES LTD.
}

1-1 Kamikodanaka 4-Chome, Nakahara-ku, Kawasaki 211-88, Japan

\begin{abstract}
In this paper we report one successful application of Quantifier Elimination $(\mathrm{QE})$ method to control problems; We focus on the Semidefinite Programming (SDP) problems, which are the central among the generic Linear Matrix Inequality (LMI) problems. Since many control problems and design specifications are reduced to LMI formulations, the LMI problems are that of great practical and theoretical interest in control theory. Though the SDP problems are usually solved as convex optimization problem numerically, when we take the real parametric uncertainties into account, the SDP problems often become non-convex and most of existing numerical methods fails. Hence we present a new symbolic method based on QE and show some experiments by using existing QE package to demonstrate the capability of the method. The method gives us exact solutions and enables us to deal with non-convex and also parametric case. Moreover, by this method, model or parameter uncertainties are easy to incorporate in the SDP problems.
\end{abstract}

\section{Introduction}

Computer Algebra has been recognized as an important tool in many engineering disciplines and continues to find new fields of applications. In this paper we report one successful application of Quantifier Elimination (QE) method to a control problem.

Several interesting control system design and analysis problems can be reduced to quantifier elimination problems as shown in the followings;

- In 1975, Anderson et.al. [2]

Application of Tarski-Seidenberg decision theory ([14],[11]) to the solution of the static output feedback stabilization problem,

- In 1995, Dorato et.al.[6] / in 1996 Abdallah et.al. [1]

Application of QE theory to a robust multi-objective design for linear systems (stability, robust stability, robust performance), 
- In 1996, Jirstrand [9]

Application of QE theory to linear systems (stabilization, feedback design) and nonlinear systems (computation of stationary points and curve following in the state space).

The first attempt to reduce some control problems to QE problems by Anderson et al. [2] was made in 1970's. But at that time the algorithm of QE was very intricate and no appropriate software was available. However, recently some improved algorithms have been developed (see [4],[5],[16], [18]) and implemented on computers (see [8],[12],[13]). By virtue of the considerable developments of both algorithms and software in QE methods, we explore the application of the QE theory to control problems of great practical interest.

In this paper, we focus on the Semidefinite Programming (SDP) problems, which are one of the generic Linear Matrix Inequality (LMI) problems. The SDP problems are usually solved as convex optimization problem numerically (see [3],[17]). When we consider the real parametric uncertainties the problems are not always convex (often become non-convex) and most of such methods does not work. So it is desirable to develop the methods which also work for non-convex case and parametric case.

In this paper we present a new symbolic method based on QE for the SDP problems and show some experiment by using existing QE package to demonstrate the capability of the method. Though currently this method is practically applicable to modest size problems which existing QE softwares can solve, it gives us exact solutions and enables us to deal with non-convex and also parametric case. Moreover, in this method, model or parameter uncertainties are easy to incorporate in the SDP problems.

\section{LMI and SDP}

Many control problems and design specifications are reduced to LMI formulations (see $[3],[7])$. Hence the LMI problems are that of great practical and theoretical interest in control theory. Here we review the definitions of LMI and the SDP problems.

A symmetric matrix $A \in \mathbf{R}^{n \times n}$ is (semi) positive definite if and only if quadratic forms $x^{T} A x>0(\geq 0)$ for all $x=\left(x_{1}, \cdots, x_{n}\right) \in \mathbf{R}^{n}$ s.t. $x \neq 0$, where $x^{T}$ stands for transpose of $x$. In the sequel, when $A$ is (semi) positive definite, we denote it by $A>_{d} 0\left(\geq_{d} 0\right)$.

Remark 1 For a real symmetric matrix $A, A>_{d} 0\left(\geq_{d} 0\right)$ if and only if all eigenvalues of $A$ are positive (non negative).

Definition 1 A linear matrix inequality (LMI) is a matrix inequality of the form

$$
F(x)=F_{0}+\sum_{i=1}^{m} x_{i} F_{i}>_{d} 0\left(\geq_{d} 0\right)
$$

where $x \in \mathbf{R}^{m}$ is the variable vector and $F_{i}=F_{i}^{T} \in \mathbf{R}^{n \times n}, i=0, \cdots, m$, are the symmetric matrices. 
SDP problems: In general, there are three types of generic LMI problems; Feasibility problem, Linear objective minimization problem under LMI constraints and Generalized eigenvalue minimization problem (see [7]). Among them we consider the problem of minimizing a linear objective function in a vector variable $x \in \mathbf{R}^{m}$ subject to a linear matrix inequality $F(x)$,

$$
\begin{array}{ll}
\text { minimize } & c^{T} x \\
\text { subject to } & F(x) \geq_{d} 0,
\end{array}
$$

where $c \in \mathbf{R}^{m}$. This problem is called Semidefinite Programming(SDP). For a vector $x_{0}$, if $F\left(x_{0}\right) \geq_{d} 0, x_{0}$ is called feasible. If there are no feasible solution, we say that the problem (2) infeasible. Note the optimal solution is on the boundary of the (convex) feasible set. SDP includes many important optimization problems (em e.g., linear and quadratic programming) as special cases and finds many applications in engineering and combinatorial problems.

\section{Quantifier Elimination}

Many mathematical and industrial problems can be translated to formulas consisting of polynomial equations, inequalities, quantifiers $(\forall, \exists)$ and Boolean operators $(\wedge, \vee, \neg, \rightarrow$ ,etc). Such formulas construct sentences in the so-called first-order theory of real closed fields and are called first-order formulas.

Let $f_{i}(X, U) \in \mathbf{Q}[X, U], i=1,2, \cdots, t$, where $\mathbf{Q}$ is the fields of rational numbers, $X=$ $\left(x_{1}, \cdots, x_{n}\right) \in \mathbf{R}^{n}$ a vector of quantified variables, and $U=\left(u_{1}, \cdots, u_{m}\right) \in \mathbf{R}^{m}$ a vector of unquantified parameter variables. Let $F_{i}=f_{i}(X, U) \square_{i} 0$, where $\square_{i} \in\{=, \geq,>, \neq\}$, for $i=1, \cdots, s, Q_{j} \in\{\forall, \exists\}$, and $X_{j}$ a block of $q_{j}$ quantified variables for $j=1, \cdots, s$. In general, quantified formula $\varphi$ is given as follows;

$$
\varphi=\left({ }^{Q_{1}} X_{1} \ldots{ }^{Q_{s}} X_{s}\right) G\left(F_{1}, \cdots, F_{t}\right)
$$

where $G\left(F_{1}, \cdots, F_{t}\right)$ is a quantifier-free (qf) Boolean formula.

$\mathrm{QE}$ procedure is an algorithm to compute equivalent $\mathrm{qf}$ formula for a given first-order formula. If all variables are quantified, i.e. $m=0, \mathrm{QE}$ procedure decides whether the given formula (3) is true or false. This problem is called decision problem. When there are some unquantified variables $U, \mathrm{QE}$ procedure find a ff formula $\varphi(U)$ describing the range of possible $U$ where $\varphi(U)$ is true. If there is no such range $\mathrm{QE}$ outputs false. This problem is called general quantifier elimination problem.

The history of the algorithms for QE begins with Tarski-Seidenberg decision procedure in 1950's [14], [11]. But this is very intricate and far from feasible. In 1975, Collins presented a more efficient general purpose QE algorithm based on Cylindrical Algebraic Decomposition (CAD) [4]. The algorithm has improved by Collins and Hong in [5] and was implemented on SACLIB as "QEPCAD" by Hong. Weispfenning also presented a very efficient $\mathrm{QE}$ algorithm based on test terms [15],[16],[18]. Though there is some degree 
restriction of a quantified variable in input formulas for test terms approach, this approach seems very practical. Implementation of the method was done on Reduce as "REDLOG" and Risa/Asir ${ }^{1)}$ [10] by Sturm [12], [13]. In this paper we use both QE implementation on Risa/Asir and REDLOG.

\section{Solving SDP by QE}

In this section we show how SDP problems are reduced to QE problems and how those are solved by using QE techniques.

\subsection{Reducing SDP to QE problems}

Determining (semi)definiteness for a real symmetric matrix is achieved without computing eigenvalues of the matrix by using the following well-known as Sylvester's theorem; For a matrix $A \in \mathbf{R}^{n \times n}$, we denote by

$$
A\left(\begin{array}{cccc}
i_{1} & i_{2} & \cdots & i_{r} \\
j_{1} & j_{2} & \cdots & j_{r}
\end{array}\right)
$$

the $r \times r$ submatrix of $A$ which consists of $\left(i_{k}, j_{l}\right)$-entries of $A$, where $1 \leq i_{1}<i_{2}<\cdots<$ $i_{r} \leq n$ and $1 \leq j_{1}<j_{2}<\cdots<j_{r} \leq n$.

Theorem 1 (Sylvester's criterion) Let $A=\left(a_{i j}\right) \in \mathbf{C}^{n \times n}$ be a Hermitian matrix. Then

(i) $A$ is positive semi-definite if and only if all principal minors of $A$ are non negative i.e.

$$
\operatorname{det} A\left(\begin{array}{cccc}
i_{1} & i_{2} & \cdots & i_{r} \\
i_{1} & i_{2} & \cdots & i_{r}
\end{array}\right) \geq 0
$$

for $1 \leq i_{1}<i_{2}<\cdots<i_{r} \leq n, r=1,2, \cdots, n$.

(ii) $A$ is positive definite if and only if all leading principal minors of $A$ are positive i.e.

$$
\operatorname{det} A\left(\begin{array}{llll}
1 & 2 & \cdots & r \\
1 & 2 & \cdots & r
\end{array}\right)>0 \quad \text { for } r=1,2, \cdots, n \text {. }
$$

This is the key to reduce SDP problems to QE problems. By Theorem 1 (i), $A(x) \geq_{d} 0$ can be reduced to the formula which is the conjunction of $2^{n}-1\left(\equiv \sum_{r=1}^{n}{ }_{n} C_{r}\right)$ inequalities.

Remark 2 By this theorem, Positive-Definite Programming can be also resolved in the same manner as SDP problems if we use (ii).

\subsection{Optimization by QE and SDP}

By Sylvester's criterion semidefinite constraints are reduced to a conjunction of inequalities and the SDP problems are reduced to the ordinary nonlinear programming problems.

\footnotetext{
1) Risa/Asir is a computer algebra system developed at Fujitsu Labs Ltd. FTP:endeavor.fujitsu.co.jp:/pub/isis/asir
} 
In [19] Weispfenning showed that the optimization problem; in particular linear programming problems, can be solved successfully by using his highly improved QE algorithm. We utilize his algorithm for a nonlinear programming problem derived from the SDP problem as above. This explains how we solve the SDP problems by using QE.

Here we show the brief sketch of his method to solve optimization problems given by a Boolean system $\psi(X, U)$ consisting of equations and inequalities and an objective function $h(X, U)$; First introduce a new indeterminate $z$ assigned to the object function $h$. Consider the new Boolean system $\psi^{\prime}=\psi \wedge(z-h \geq 0)$. Then the problem minimizing $h$ subject to $\psi(X, U)$ is formulated as first-order formula $\varphi={ }^{\exists} x_{1} \ldots{ }^{\exists} x_{n}\left(\psi^{\prime}\right)$. Next eliminate all quantified variables $x_{1}, \cdots, x_{n}$ to have the resulting qf formula $\varphi^{\prime}$ in $z$ and $U$. After that we specialize parameters $U$ of $\varphi^{\prime}$ by an appropriate real values, then $\varphi^{\prime}$ gives a finite union $M$ of intervals for $z$, which shows a possible range of $z$. If $M$ is empty, $\psi$ is unsolvable (i.e. infeasible); if $M$ is unbounded from below, $h$ has no minimum w.r.t. $\psi$; if $m \in M$ is a lowest endpoint of $M$, then $m$ is the minimum value of $z$ w.r.t. $\psi$ (for details [19]). As for the coordinates of a minimum point for $z$ can be obtained by back-substitution from the test terms used during the elimination if we use test term approach (see [19]).

Remark 3 Results of $Q E$ are exact but usually large, particularly in parametric case. In the parametric case, in general, results are of the form $\bigvee$ (case distinctions $\wedge$ possible range of $z$ ). Then if we specialize the parameters of the results, we obtain the desired possible range of $z$.

\section{Examples}

We show some examples (which are modified one taken from [17]), and apply the above mentioned method to them in order to demonstrate the potential of QE approach to SDP problems. The following experiments were done by "Risa/Asir" on a PC with P6-200MHz $\mathrm{CPU}$, and all results are obtained immediately.

- Non-Parametric: Minimize $x_{1}+x_{2}$ subject to

$$
\left[\begin{array}{ccc}
1 & x_{1} & 0 \\
x_{1} & x_{2} & 0 \\
0 & 0 & x_{1}+1
\end{array}\right] \geq_{d} 0
$$

The semidefinite constraint (6) is reduced to a Boolean system of inequalities constraints by using Sylvester's criterion;

$$
\begin{gathered}
\psi=(1 \geq 0) \wedge\left(x_{2} \geq 0\right) \wedge\left(x_{1}+1 \geq 0\right) \wedge\left(x_{2}-x_{1}^{2} \geq 0\right) \wedge\left(x_{2} x_{1}+x_{2} \geq 0\right) \wedge \\
\left(x_{1}+1 \geq 0\right) \wedge\left(-x_{1}^{3}-x_{1}^{2}+x_{2} x_{1}+x_{2} \geq 0\right)
\end{gathered}
$$

Assign new slack variable $z$ to objective function $x_{1}+x_{2}$ and let $\psi^{\prime}=\psi \wedge\left(z-x_{1}-x_{2} \geq 0\right)$. Then the problem is formulated as a first-order formula $\varphi={ }^{\exists} x_{1}{ }^{\exists} x_{2}\left(\psi^{\prime}\right)$. After QE we 
have an equivalent qf formula describing the range of objective function;

$$
4 z+1 \geq 0
$$

- Parametric[1]: Minimize ax $x_{1}+x_{2}$ subject to (6) with a parameter a.

Let $\psi_{P_{1}}^{\prime}=\psi \wedge\left(z-a x_{1}-x_{2} \geq 0\right)$. The problem is formulated as $\varphi_{P_{1}}={ }^{\exists} x_{1}{ }^{\exists} x_{2}\left(\psi_{P_{1}}^{\prime}\right)$. After $\mathrm{QE}$ we have an equivalent qf formula describing the range of objective function $z$ with a parameter $a$;

$$
\begin{gathered}
(a+z-1 \geq 0) \vee(z \geq 0) \vee \\
\left(\left(a^{3}-2 a^{2} z+a^{2}+a z^{2}-a z \leq 0\right) \wedge\left(a^{2}-a z \geq 0\right) \wedge(a-z+1 \leq 0) \wedge(a=0)\right) \vee \\
\left(\left(a^{3}-a^{2}+3 a z-2 z \leq 0\right) \wedge\left(a^{2}+4 z \geq 0\right) \wedge\left(a^{2}+2 z \geq 0\right) \wedge(a-2 \leq 0)\right) \vee \\
\left(\left(a^{2}+4 z \geq 0\right) \wedge(a-2<0)\right) .
\end{gathered}
$$

If we substitute a parameter $a$ with 1 and simplify the result, we have same result as (7).

- Restricted region: Minimize $x_{1}+x_{2}$ subject to (6) in restricted domains $D_{1}, D_{2}$ respectively, where (i) $D_{1}=\left\{x_{1}, x_{2} \in \mathbf{R} \mid 0 \leq x_{1} \leq 3,0 \leq x_{2} \leq 5\right\}$ and (ii) $D_{2}=\left\{x_{1}, x_{2} \in\right.$ $\left.\mathbf{R} \mid 0 \leq x_{1} \leq 10,5 \leq x_{2} \leq 10\right\}$.

(i) In this case the problem is formulated as

$$
\varphi_{D_{1}}={ }^{\exists} x_{1}{ }^{\exists} x_{2}\left(\psi^{\prime} \wedge\left(0 \leq x_{1} \leq 3 \wedge 0 \leq x_{2} \leq 5\right)\right)
$$

After QE we have $4 z+1 \geq 0$.

(ii) In this case the problem is formulated as

$$
\varphi_{D_{2}}={ }^{\exists} x_{1}{ }^{\exists} x_{2}\left(\psi^{\prime} \wedge\left(0 \leq x_{1} \leq 10 \wedge 5 \leq x_{2} \leq 10\right)\right)
$$

After QE we have

$$
\begin{gathered}
(z-4 \geq 0) \vee\left(\left(z^{2}-10 z+20 \leq 0\right) \wedge\left(z^{2}-20 z+90 \geq 0\right) \wedge(4 z+1 \geq 0) \wedge(z \leq 0)\right) \vee \\
\left(\left(z^{2}-10 z+20 \leq 0\right) \wedge\left(z^{2}-20 z+90 \geq 0\right) \wedge(4 z+1 \geq 0) \wedge(z<0)\right)
\end{gathered}
$$

- Parametric[2]: Minimize $x_{1}+x_{2}$ subject to

$$
\left[\begin{array}{ccc}
s & x_{1} & 0 \\
x_{1} & x_{2} & 0 \\
0 & 0 & x_{1}+1
\end{array}\right] \geq_{d} 0
$$


where $s$ is a parameter.

The semidefinite constraint (8) is reduced to

$$
\begin{gathered}
\psi_{P}=(s \geq 0) \wedge\left(x_{2} \geq 0\right) \wedge\left(x_{1}+1 \geq 0\right) \wedge\left(s x_{2}-x_{1}^{2} \geq 0\right) \wedge \\
\left.\left(x_{2} x_{1}+x_{2}\right) \geq 0\right) \wedge\left(s\left(x_{1}+1\right) \geq 0\right) \wedge\left(\left(x_{2} x_{1}+x_{2}\right) s-x_{1}^{3}-x_{1}^{2} \geq 0\right)
\end{gathered}
$$

Let $\psi_{P_{2}}^{\prime}=\psi_{P} \wedge\left(z-x_{1}-x_{2} \geq 0\right)$. Then the problem is formulated as a first-order formula $\varphi_{P_{2}}={ }^{\exists} x_{1}{ }^{\exists} x_{2}\left(\psi_{P_{2}}^{\prime}\right)$. After QE we obtain

$(s z+s-1 \geq 0 \wedge s>0 \wedge z+1 \geq 0) \vee(s z+s-1 \geq 0 \wedge s \geq 0 \wedge z+1 \geq 0) \vee(s z+s \geq 0 \wedge s \geq$ $0 \wedge z=0) \vee\left(s^{2}+4 s z \geq 0 \wedge\left(\left(s^{2}-2 s \leq 0 \wedge s z+s-1 \leq 0 \wedge s-2 \leq 0 \wedge s \geq 0 \wedge\left(s^{2}+3 s z-s-2 z \leq\right.\right.\right.\right.$ $0 \vee(s+z-1 \leq 0 \wedge(s z+s-1=0 \vee z=0))) \wedge(s+2 z \geq 0 \vee z=0)) \vee\left(s+2 z \geq 0 \wedge s \geq 0 \wedge\left(s^{2}-2 s \leq\right.\right.$ $0 \vee s z+s-1 \geq 0 \vee s=0) \wedge\left(s^{2}-2 s \leq 0 \vee s>0\right) \wedge\left(\left(s^{2}+3 s z-s-2 z \leq 0 \wedge(s z+s-1 \leq 0 \vee z=\right.\right.$ $0)) \vee(s+z-1 \geq 0 \wedge(s z+s-1 \geq 0 \vee z=0))) \wedge(s z+s-1 \geq 0 \vee s-2 \leq 0)))) \vee(s \geq 0 \wedge z \geq$ $0) \vee\left(s^{2}-2 s \leq 0 \wedge s+4 z \geq 0 \wedge s-2 \leq 0 \wedge s>0\right) \vee\left(s^{2} z+s^{2}-s \geq 0 \wedge s>0\right) \vee\left(s^{2}-2 s \leq 0 \wedge s+4 z \geq\right.$ $0 \wedge s-2<0 \wedge s>0) \vee(s>0 \wedge z \geq 0) \vee\left(s>0 \wedge\left(s^{2} z+s^{2}-s>0 \vee\left(s^{2}-2 s<0 \wedge s z+s-1=0\right)\right)\right)$.

If we substitute the parameter $s$ with 1 and simplify the result, we have same result as (7).

- Parameter uncertainty: Minimize $x_{1}+x_{2}$ subject to (8) within the regions of a parameter $s$ (i) $-5 \leq s \leq-1$, (ii) $-10 \leq s \leq 0$, respectively.

For (i),(ii), the problem is formulated as a first-order formula; $\varphi_{(i)}={ }^{\exists} x_{1}{ }^{\exists} x_{2}\left(\psi_{P_{2}}^{\prime} \wedge(-5 \leq\right.$ $s \leq-1)), \varphi_{(i i)}={ }^{\exists} x_{1}{ }^{\exists} x_{2}\left(\psi_{P_{2}}^{\prime} \wedge(-10 \leq s \leq 0)\right)$, respectively. After QE we obtain "false" for (i) and for (ii)

$(s z+s-1 \geq 0 \wedge s=0 \wedge z+1 \geq 0) \vee(s z+s \geq 0 \wedge s=0 \wedge z=0) \vee\left(s^{2}+4 s z \geq 0 \wedge\left(\left(s^{2}-2 s \leq\right.\right.\right.$ $0 \wedge s z+s-1 \leq 0 \wedge s=0 \wedge\left(s^{2}+3 s z-s-2 z \leq 0 \vee(s+z-1 \leq 0 \wedge(s z+s-1=0 \vee z=\right.$

$0))) \wedge(s+2 z \geq 0 \vee z=0)) \vee\left(s^{2}-2 s \leq 0 \wedge s+2 z \geq 0 \wedge s=0 \wedge\left(\left(s^{2}+3 s z-s-2 z \leq\right.\right.\right.$ $0 \wedge(s z+s-1 \leq 0 \vee z=0)) \vee(s+z-1 \geq 0 \wedge(s z+s-1 \geq 0 \vee z=0)))))) \vee(s=0 \wedge z \geq 0)$.

\section{Concluding Remarks}

We showed that the SDP problems, which is one of the greatly important problems in LMI problems, are reduced to quantifier elimination problems by Sylvester's criterion and presented the concrete procedure to solve SDP problems by using QE for some examples.

The efficiency of the method proposed here depend on deeply that of quantifier elimination procedure. Due to the great theoretical and practical complexity of quantifier elimination for the elementary theory of the real closed field, currently the method is practically applicable to moderate size problems for general input formulas or considerable large size problems for special type input formulas.

However, the method based on QE has many merits listed below compared with existing (numerical) methods for SDP problems; 
- Non-convex case can be dealt with.

- Parametric case can be dealt with.

When we consider parametric uncertainties, LMI problems often become non-convex. This is not an issue since QE does not utilize any information about convexity of the problem.

- Easy to handle the domain restricted case and parameter uncertainty.

What we do is just add conjunctively the corresponding constraint to the original formula.

- Produce exact answer and check the infeasibility exactly.

"exact answer" also implies that this method leaves no hole in the parameter space in the deterministic discretization case and yields probability-one results in the random discretization case [1].

Hence, QE methods may provide the solutions for some problems that would be difficult to obtain by existing methods.

Acknowledgments The author would like to thank Prof. V. Weispfenning (Univ. of Passau) and his colleagues J. Kaneko and K. Yokoyama for their invaluable comments and advice. And he also would like to thank T. Sturm (Univ. of Passau) for his cooperation about implementation and experiments.

\section{参 考 文 献}

[1] Abdallah, C., Dorato, P., Yang, W., Liska, R., Steinberg, S. (1996). Application of Quantifier Elimination Theory to Control System Design. Proceedings 4th IEEE Mediterranean Symposium on Control and Automation. Maleme, Crete, pp340-345.

[2] Anderson, B., Bose, N., Jury, E. (1975). Output feedback stabilization and related Problems - solution via decision methods. IEEE Trans. Auto. Control, pp 53-65.

[3] Boyd, S., Ghaoui, L,E., Feron, E,. Balakrishnan, V. (1994). Linear Matrix Inequalities in System and Control Theory. SIAM Studies in Applied Mathematics, vol 15.

[4] Collins, G,E. (1975). Quantifier Elimination in the elementary theory of real closed fields by cylindrical algebraic decomposition for quantifier elimination. LNCS 33, 134-183, SpringerVerlag, Berlin.

[5] Collins, G,E., Hong, H. (1991). Partial cylindrical algebraic decomposition for quantifier elimination. J. Symb. Comp. 12, No.3, pp299-328.

[6] Dorato, P., Yang, W., Abdallah, C. (1995). Application of Quantifier Elimination Theory to Robust Multi-object Feedback Design. J. Symb. Comp. 11 pp1-6.

[7] Gahinet, P., Nemirovski, A., Laub, A.J., Chilai, M. (1995). LMI Control Toolbox User's Guide, For Use with MATLAB. The MATH WORKS INC. 
[8] Hong, H. (1992). Simple solution formula construction in cylindrical algebraic decomposition based quantifier elimination. ISSAC'92, International Symposium on Symbolic and Algebraic Computation. ACM Press, pp177-188.

[9] Jirstrand, M. (1996). Algebraic Methods for Modeling and Design in Control. Linköping Studied in Science and Technology, Thesis No. 540.

[10] Noro, M., Takeshima, T. (1992). Risa/Asir - a computer algebra system, in "Proc. ISSAC '92," ACM Press, pp. 387-396.

[11] Seidenberg, A. (1954). A new decision method for elementary algebra. Annals of Math., vol. 60 , pp. 365-374.

[12] Sturm, T. (1994). REDLOG, reduce library of algorithms for manipulation of first-order formulas. Univ. of Passau, Technical Report.

[13] Sturm, T. (1996). Real Quadratic Quantifier Elimination in Risa/Asir. Fujitsu Labs Research Report ISIS-RR-96-13E.

[14] Tarski, A. (1951). Decision Methods for Elementary Algebra and Geometry. Berkeley: Univ. of California Press.

[15] Weispfenning, V. (1988). The complexity of linear problems in fields. J. Symb. Comp. 5,(1), 3-27.

[16] Loos, R., Weispfenning, V. (1996). Applying linear Quantifier elimination. The Computer Journal, Vol.36, No.5. pp450-462.

[17] Vandenberghe, L., Boyd, S. (1996). Semidefinite Programming. SIAM Review, March, Vol.38, No.1, pp 49-95

[18] Weispfenning, V. (1996). Quantifier Elimination for real algebra - the quadratic case and beyond. To appear in AAECC, 1996.

[19] Weispfenning, V. (1996). Simulation and Optimization by Quantifier Elimination. J. Symb. Comp. to appear. 